Canadian

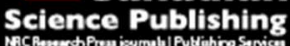

Applied Physiology, Nutrition, and Metabolism Physiologie appliquée, nutrition et métabolisme

\title{
Short-term remote ischemic preconditioning is not associated with improved blood pressure and exercise capacity in young adults
}

\begin{tabular}{|r|l|}
\hline Journal: & Applied Physiology, Nutrition, and Metabolism \\
\hline Manuscript ID & apnm-2016-0024.R2 \\
\hline Manuscript Type: & Brief communication \\
\hline Date Submitted by the Author: & $30-$ Mar-2016 \\
\hline Complete List of Authors: & $\begin{array}{l}\text { Banks, Laura; Hospital for Sick Children } \\
\text { Wells, Greg; Hospital for Sick Children } \\
\text { Clarizia, Nadia; The Hospital for Sick Children, Cardiology } \\
\text { Jean-St-Michel, Emilie; The Hospital for Sick Children, Cardiology } \\
\text { McKillop, Adam; The Hospital for Sick Children, Cardiology - CVCRU } \\
\text { Redington, Andrew; Hospital for Sick Children, Cardiology; University of } \\
\text { Toronto, Pediatrics } \\
\text { McCrindle, Brian; Hospital for Sick Children }\end{array}$ \\
\hline Keyword: & $\begin{array}{l}\text { magnetic resonance spectroscopy, ischemia-reperfusion, phosphocreatine } \\
\text { recovery, cardiac energy metabolism, peak oxygen consumption }\end{array}$ \\
\hline \multicolumn{2}{|c}{} \\
\hline
\end{tabular}


Short-term remote ischemic preconditioning is not associated with improved blood pressure and exercise capacity in young adults

Banks, Laura ${ }^{1}, \mathrm{PhD}$, Wells, Greg D. ${ }^{2,4}$, PhD, Clarizia, Nadia A. ${ }^{1}, \mathrm{MD}$, Jean-St-Michel, Emilie ${ }^{1}$, MD, McKillop, Adam L. ${ }^{1}$, MSc, Redington, Andrew N. ${ }^{1,3}$, MD, McCrindle, Brian $\mathrm{W}^{1,3}, \mathrm{MD}, \mathrm{MPH}$.

${ }^{1}$ Labatt Family Heart Centre, Hospital for Sick Children, Toronto, Ontario, Canada

${ }^{2}$ Faculty of Kinesiology and Physical Education, University of Toronto, Toronto, Ontario, Canada

${ }^{3}$ Department of Pediatrics, University of Toronto, Toronto, Ontario, Canada

${ }^{4}$ Physiology and Experimental Medicine, the Hospital for Sick Children, Toronto, Ontario, Canada

Corresponding Author: Dr. Brian McCrindle

Hospital for Sick Children

555 University Avenue

Toronto, Ontario, M5G 1X8

Phone: 416-813-7654 ext. 207609

Email: brian.mccrindle@sickkids.ca

Funding Source: Canadian Institutes of Health Research (CIHR)

Word Count: 2461 (excluding 2 Figures) 


\section{Abstract}

Purpose: We sought to determine whether a 9-day remote ischemic preconditioning (IPC) causes improvements in exercise performance, energetics, and blood pressure.

Results: Ten participants (mean age $=24 \pm 4$ years) had no changes in aerobic capacity (pre-intervention: $38 \pm 10 \mathrm{ml} / \mathrm{kg} / \mathrm{min}$ vs. post-intervention: $38 \pm 10 \mathrm{ml} / \mathrm{kg} / \mathrm{min}$ ), blood pressure (pre-intervention: $112 \pm 7 / 66 \pm 6 \mathrm{mmHg}$ vs. post-intervention: $112 \pm 10 / 62 \pm 5 \mathrm{mmHg}$ ), cardiac phosphocreatinine-to-adenosine-triphosphate ratio (preintervention: $2.1 \pm 0.5$ vs. post-intervention: $2.3 \pm 0.4$ ), and post-exercise skeletal muscle phosphocreatine recovery (pre-intervention: $34 \pm 11$ seconds vs. post-intervention: $31 \pm 11$ seconds).

Conclusions: Short-term remote IPC may be ineffective in improving these outcomes.

Keywords: ischemia-reperfusion, peak oxygen consumption, phosphocreatine recovery, cardiac energy metabolism, magnetic resonance spectroscopy 
Introduction

Ischemia-reperfusion injury is common in many cardiovascular diseases. Remote ischemic preconditioning (IPC) can be induced by nonlethal ischemia at a site distant to the target organ (e.g., using a blood pressure cuff or tourniquet to occlude blood flow in a limb) before a prolonged ischemic episode. Acute remote IPC has been shown to provide protection against subsequent ischemia-reperfusion injury of distant organs in humans(Przyklenk et al. 1993).

A growing number of studies have more recently examined the effectiveness of remote IPC on blood pressure, exercise performance, and muscle energetics in adults(Jean-St-Michel et al. 2011; Madias 2011; Clevidence et al. 2012; Jones et al. 2014; Lalonde and Curnier 2014). Early evidence suggests that mean arterial and/or systolic blood pressure may be improved with acute and chronic remote IPC(Madias 2011; Jones et al. 2014). In contrast, acute remote IPC may or may not effect aerobic and anaerobic performance(Jean-St-Michel et al. 2011; Clevidence et al. 2012; Lalonde and Curnier 2014; Incognito et al. 2015). Ischemic preconditioning may conceivably modulate muscle energetics during reperfusion, as evidenced by an increase in phosphocreatine production as well as higher oxygen consumption(Andreas et al. 2011). The effectiveness of chronic remote IPC on improving blood pressure and exercise performance warrants further investigation.

As a result, we sought to determine the influence of a short-term remote IPC intervention on blood pressure, peak exercise performance, and muscle energetics. It was hypothesized that the whole-body effects of a 9-day remote IPC intervention would 
be associated with a decrease in resting blood pressure and an increase in aerobic exercise capacity secondary to enhanced muscle energetics.

Materials and Methods

Recruitment: Institutional research ethics board approval was obtained. Sedentary young adults were recruited from the local community and informed consent was obtained. Potential participants (18-35 year old sedentary males and females) were excluded if there was a contraindication to exercise or magnetic resonance imaging, or a prior cardiovascular disease diagnosis. Study participation was delayed for the following reasons: reported vigorous exercise (21 days prior to the IPC intervention), and/or an acute viral or bacterial infection (10 days prior to the IPC intervention), and/or alcohol and/or caffeine consumption (10 days prior to the intervention or during the study period). These rigorous criteria reflected factors that were known to have potential preconditioning effects and/or mitigate the effects of preconditioning. Adherence was confirmed verbally and recorded at each study visit.

Remote IPC Intervention: A remote IPC protocol was performed at the same time daily (morning) for 9 consecutive days. Following a 5-minute rest period, an automated blood pressure (BP) device (General Electric Dash 3000 Monitor, WI, USA) was used to assess seated blood pressure in triplicate before and after each intervention day. Remote IPC was induced using a standard hand anaeroid sphygmomanometer and blood pressure cuff placed on the right arm of the seated participant. A consecutive day intervention protocol was chosen to coincide with established pre-conditioning windows. 
The blood pressure cuff was placed on the upper arm, as it has been shown to be more tolerable and easier to occlude than the lower limb(Clevidence et al. 2012). This site was also considered suitable as preconditioning is known to have whole-body effects, particularly at sites distant from the application of the blood pressure cuff. The cuff was inflated to a pressure of $200 \mathrm{mmHg}$ for five minutes (ischemia). The blood pressure cuff was then deflated for five minutes (reperfusion) completing one cycle of ischemiareperfusion. A total of four inflation and deflation cycles were performed daily.

Cardiopulmonary Exercise Testing: A progressive ramp bicycle exercise test was performed to determine peak oxygen consumption (peak $\mathrm{VO}_{2}$ )one week prior to and at least $24 \mathrm{~h}$ following the 9-day remote IPC intervention (Godfrey et al. 1971). This cycle ergometry (Lode Corval, Lode BV Medical Technology, Groningen, NL) protocol involved 1-minute increments of 10-20 Watts (dependent on participant height) to determine peak $\mathrm{VO}_{2}$ within a 10-12 minute exercise test. Blood pressure (Tango, Suntech Medical, NC, USA) and lactate measures (iSTAT 300 with CG4+ Cartridges, Abbott Laboratories, IL, USA) were taken at rest and peak exercise. This gold-standard measure also included the breath-by-breath analysis of oxygen consumption $\left(\mathrm{VO}_{2}\right)$, respiratory exchange ratio, and heart rate (Vmax Encore with Cardiosoft ECG v6.51, Viasys Healthcare, CA, USA). Anaerobic threshold was determined following the test using the V-slope method.(Beaver et al. 1986) The ratio of whole body mass to height squared was calculated to determine body mass index (BMI in $\mathrm{kg} / \mathrm{m}^{2}$ ). 
${ }^{31}$ Phosphorus-Magnetic Resonance Spectroscopy ( $\left.{ }^{31} \mathrm{P}-\mathrm{MRS}\right):{ }^{31} \mathrm{P}-\mathrm{MRS}$ data were collected to examine cardiac and skeletal muscle energetics according to a standard, reproducible protocol(Banks et al. 2014). Data were collected at baseline (10 days prior to the study intervention), on days 1 and 2 of the study intervention (following the acute remote IPC bout), and following the study intervention (24 hours following the last day of the RIPC intervention. The inorganic phosphate-to-phosphocreatine ratio (Pi/PCr) was calculated to provide an estimate of ADP concentration (e.g. energy depletion). $\mathrm{PCr}$ recovery half-time was calculated to provide an estimate of oxidative capacity in skeletal muscle. Resting cardiac muscle PCr/ATP was calculated to provide an estimate of cardiac energy reserve..

Statistical Analysis: All statistical analyses were performed using SAS v.9.2 (The SAS Institute, Cary NC). Rejection of the null hypothesis was set a priori at an alpha level of 0.05 . Descriptive statistics were performed to establish baseline participant characteristics (reported as mean \pm standard deviation). Linear regression analysis (with maximum likelihood algorithm; reported as parameter estimate[standard error]) was performed to examine changes in primary study outcomes with the intervention.

Results

Subject Characteristics: Participants ( $n=10 ; 4$ males, 6 females; mean age $=24 \pm 4$ years, $B M I=25.2 \pm 4.1 \mathrm{~kg} / \mathrm{m}^{2}$, mean resting systolic $B P /$ diastolic $B P$ : 
$112 \pm 7 / 66 \pm 6 \mathrm{mmHg}$ ) completed nine consecutive days of the IPC intervention with $100 \%$ compliance.

Blood Pressure (BP): BP was unchanged following the 9-day IPC intervention (systolic BP: $-0.1[0.4] \mathrm{mmHg}, p=0.77$; diastolic BP: $-0.1[0.2] \mathrm{mmHg}, p=0.72$ ). Individual changes in systolic BP are shown in Figure 1.

Cardiopulmonary Exercise Testing: Exercise data are summarized in Table 1. Briefly, pre-intervention hemodynamic and metabolic variables were within a normal healthy range. Anaerobic threshold and peak $\mathrm{VO}_{2}$ were unchanged following the intervention. Individual changes in peak $\mathrm{VO}_{2}$ are shown in Figure 2. Furthermore, the RIPC intervention was not associated with secondary measures, including a decrease in resting lactate $(-0.3[0.3] \mathrm{mmol} / \mathrm{L}, \mathrm{p}=0.25)$, increase in peak heart rate $(5.3[3.9]$ beats per minute, $p=0.18)$, and increase in peak lactate $(1.6[1.1] \mathrm{mmol} / \mathrm{L}, \mathrm{p}=0.16)$, after controlling for peak exercise power output.

Cardiac and Skeletal Muscle Energetics: ${ }^{31} \mathrm{P}-\mathrm{MRS}$ results are summarized in Table 2. Briefly, cardiac muscle PCr/ATP was unchanged following the remote IPC intervention. Seven participants with similar baseline characteristics $(n=7 / 10$, mean age $=25 \pm 5$ years, $B M I=26 \pm 5 \mathrm{~kg} / \mathrm{m}^{2}$ ) performed the skeletal muscle ${ }^{31} \mathrm{P}-\mathrm{MRS}$ protocol Three participants $(n=3 / 10)$ were unable to complete the skeletal muscle ${ }^{31} \mathrm{P}-\mathrm{MRS}$ protocol due to equipment malfunction. Post-exercise Pi/PCr was increased (e.g. energy depletion) in skeletal muscle in the MRI over the nine-day intervention, while post-exercise $\mathrm{PCr}$ recovery remained unchanged. 


\section{Discussion}

This exploratory trial examined the effects of a 9-day remote IPC intervention on blood pressure, peak exercise performance, and muscle energetics in sedentary, young adults, thereby representing a novel opportunity to investigate early human adaptation to a form of relative tissue ischemia/hypoxemia. No significant changes in primary outcome measures were observed, with the exception of an increase in post-exercise energy depletion in skeletal muscle.

The effect of remote IPC on resting blood pressure: The relationship between blood pressure and remote IPC has received attention lately.(Madias 2011; Millar and McGowan 2012) A case study reported a decline in both systolic (>6 mm Hg) and diastolic (>3 mm Hg) at 30 minutes following an acute bout of remote IPC in a normotensive, middle-aged adult.(Madias 2011) The author hypothesized that repeated bouts of remote IPC might also be associated with a blood pressure reduction. A reduction in mean arterial blood pressure was in fact observed, secondary to improvements in systemic endothelial function, in a recent study of young, prehypertensive males who completed a seven-day remote IPC intervention (four cycles of five minute ischemia-reperfusion)(Jones et al. 2014). The remote IPC stimulus and age of study participants were similar to the current study; however, our participants had two additional days of IPC and a significantly lower pre-intervention resting blood pressure. A blood pressure reduction was not observed in our normotensive participants. A remarkably lower systolic blood pressure (approximately a $20-30 \mathrm{~mm} \mathrm{Hg}$ difference) in our participants, may suggest a "floor" effect for blood pressure reduction. The use of 
remote IPC as a therapeutic adjunct to traditional pharmacological treatment in hypertensive patients warrants further investigation.

The effect of remote IPC on exercise performance: A recent systematic review of 21 studies reported that time-trial performance improved in approximately two-thirds of athletes and recreationally active adults (Incognito et al. 2015). Nonetheless, evidence for remote IPC improving power output, oxygen consumption, rating of perceived exertion, blood lactate accumulation, and cardiorespiratory parameters remain unclear due to smaller sample sizes and within study heterogeneity (Incognito et al. 2015). Notably, a laboratory-based study documented that acute remote IPC did not improve aerobic or anaerobic exercise performance among twelve amateur cyclists(Clevidence et al. 2012). Our findings support the observations that remote IPC is not associated with an improvement in peak $\mathrm{VO}_{2}$, and expand upon previous results by including a short-term intervention (multiple vs. single remote IPC bouts) and studying a sedentary, normotensive cohort.

The effect of remote IPC on cardiac and skeletal muscle energetics: Local IPC has been reported to improve phosphocreatine production and increase oxygen consumption immediately following ischemia during the reperfusion recovery period(Andreas et al. 2011). To our knowledge, the potential benefits of acute and chronic remote IPC on muscle energetics have not been documented. We observed an increase in post-exercise skeletal muscle Pi/PCr following the short-term IPC intervention. This finding may actually suggest higher ADP concentration and relative energy depletion following the short-term IPC intervention. The significance of an increase in $\mathrm{Pi} / \mathrm{PCr}$ despite no change in $\mathrm{PCr}$ recovery half-time is unclear. 
Limitations: The following limitations should be considered when interpreting study results. These findings need to be substantiated with a larger sample size given the exploratory nature of our study. A larger sample size may help to identity potential sample characteristics (e.g. sex, cardiorespiratory fitness, disease) that mitigate the response to remote IPC. Study recruitment was challenging given the strict exclusion criteria and intensive commitment required by participants; yet, the remote IPC intervention was well-tolerated as evidenced by participant adherence.

Conclusion: Our pilot study findings suggest that short-term remote IPC stimulus is not associated with significant improvements in blood pressure, aerobic exercise capacity, and cardiac muscle energetics among young, normotensive adults.

Acknowledgements

The authors acknowledge Dr. Jane Schneiderman for her supervision of the exercise testing protocol. The authors acknowledge Tammy Rayner and Ruth Weiss for their technical expertise in ${ }^{31} \mathrm{P}-\mathrm{MRS}$ development and scanning for this study. The authors would also like to acknowledge Svetlana Khaikin and Nicole Barbosa for their help with coordinating the study. This study was funded by the Canadian Institute of Health Research (CIHR; Grant Number MOP102692).

Conflict of Interest 
Drs. McCrindle and Redington are shareholders of CellAegis Devices Inc. which produces the autoRIC $\circledast$ remote ischemic conditioning system. This system was not used in the current study.

\section{References}

Andreas M., Schmid A.I., Keilani M., Doberer D., Bartko J., Crevenna R., et al. 2011. Effect of ischemic preconditioning in skeletal muscle measured by functional magnetic resonance imaging and spectroscopy: a randomized crossover trial. Journal of cardiovascular magnetic resonance : official journal of the Society for Cardiovascular Magnetic Resonance. 1332.

Banks L., Wells G.D. and McCrindle B.W. 2014. Cardiac energy metabolism is positively associated with skeletal muscle energy metabolism in physically active adolescents and young adults. Applied physiology, nutrition, and metabolism = Physiologie appliquee, nutrition et metabolisme. 39(3): 363-368.

Beaver W.L., Wasserman K. and Whipp B.J. 1986. A new method for detecting anaerobic threshold by gas exchange. Journal of applied physiology. 60(6): 2020-2027.

Clevidence M.W., Mowery R.E. and Kushnick M.R. 2012. The effects of ischemic preconditioning on aerobic and anaerobic variables associated with submaximal cycling performance. European journal of applied physiology. 112(10): 36493654 .

Godfrey S., Davies C.T., Wozniak E. and Barnes C.A. 1971. Cardio-respiratory response to exercise in normal children. Clin Sci. 40(5): 419-431.

Incognito A.V., Burr J.F. and Millar P.J. 2015. The Effects of Ischemic Preconditioning on Human Exercise Performance. Sports medicine.

Jean-St-Michel E., Manlhiot C., Li J., Tropak M., Michelsen M.M., Schmidt M.R., et al. 2011. Remote preconditioning improves maximal performance in highly trained athletes. Medicine and science in sports and exercise. 43(7): 1280-1286.

Jones H., Hopkins N., Bailey T.G., Green D.J., Cable N.T. and Thijssen D.H. 2014. Seven-day remote ischemic preconditioning improves local and systemic endothelial function and microcirculation in healthy humans. American journal of hypertension. 27(7): 918-925. 
Lalonde F. and Curnier D. 2014. Can Anaerobic Performance Be Improved by Remote Ischemic Preconditioning? Journal of strength and conditioning research / National Strength \& Conditioning Association.

Madias J.E. 2011. Effect of serial arm ischemic preconditioning sessions on the systemic blood pressure of a normotensive subject. Medical hypotheses. 76(4): 503-506.

Millar P.J. and McGowan C.L. 2012. Serial remote ischemic preconditioning and rhythmic isometric exercise training: a hypothesis. Medical hypotheses. 78(6): 822-823.

Przyklenk K., Bauer B., Ovize M., Kloner R.A. and Whittaker P. 1993. Regional ischemic 'preconditioning' protects remote virgin myocardium from subsequent sustained coronary occlusion. Circulation. 87(3): 893-899. 
Table 1. Energy Metabolism Following Short-term Remote Ischemic

Preconditioning (IPC) Intervention

\begin{tabular}{|c|c|c|c|c|}
\hline Remote IPC Intervention & Pre & Day 1 & Day 2 & Post \\
\hline \multicolumn{5}{|c|}{ Cardiac ${ }^{31}$ Phosphorus-Magnetic Resonance Spectroscopy Results } \\
\hline PCr/ATP & $2.1 \pm 0.5$ & $2.5 \pm 0.5$ & $2.3 \pm 0.5$ & $2.3 \pm 0.4$ \\
\hline \multicolumn{5}{|c|}{ Skeletal Muscle ${ }^{31}$ Phosphorus-Magnetic Resonance Spectroscopy Results } \\
\hline $\begin{array}{l}\text { Resting phosphocreatine (PCr; } \\
\text { millimoles per liter) }\end{array}$ & $19.5 \pm 0.8$ & $20.2 \pm 1.4$ & $20.2 \pm 1.1$ & $19.6 \pm 1.4$ \\
\hline $\begin{array}{l}\text { Resting inorganic phosphate } \\
\text { (Pi; millimoles per liter) }\end{array}$ & $1.9 \pm 0.4$ & $2.1 \pm 0.5$ & $2.1 \pm 0.3$ & $2.1 \pm 0.2$ \\
\hline Resting $\mathrm{pH}$ & $6.9 \pm 0.02$ & $7.0 \pm 0.03$ & $6.9 \pm 0.02$ & $7.0 \pm 0.03$ \\
\hline $\mathrm{Pi} / \mathrm{PCr}$ (Post-30 seconds) & $0.5 \pm 0.3$ & $1.1 \pm 0.8$ & $1.2 \pm 0.5$ & $1.3 \pm 0.5$ \\
\hline Pi/PCr (Post-60seconds) & $1.0 \pm 1.0$ & $2.0 \pm 1.8$ & $2.4 \pm 2$ & $2.5 \pm 1.0$ \\
\hline $\mathrm{Pi} / \mathrm{PCr}$ (Post-2.5 minutes) & $1.0 \pm 0.7$ & $1.3 \pm 1.1$ & $2.2 \pm 1.8$ & $1.9 \pm 1.4$ \\
\hline $\begin{array}{l}\text { PCr Recovery Half-time (Post- } \\
30 \text { seconds) }\end{array}$ & $31 \pm 13$ & $33 \pm 11$ & $31 \pm 12$ & $35 \pm 10$ \\
\hline $\begin{array}{l}\text { PCr Recovery Half-time (Post- } \\
60 \text { seconds) }\end{array}$ & $34 \pm 11$ & $29 \pm 9$ & $36 \pm 8$ & $31 \pm 11$ \\
\hline $\begin{array}{l}\text { PCr Recovery Half-time (Post- } \\
2.5 \text { minutes) }\end{array}$ & $37 \pm 15$ & $36 \pm 8$ & $36 \pm 7$ & $36 \pm 9$ \\
\hline
\end{tabular}


Table 2. Cardiopulmonary Exercise Testing Data Following the Remote Ischemic Preconditioning (IPC) Intervention

\begin{tabular}{|c|c|c|}
\hline & Pre $(n=10)$ & Post $(n=10)$ \\
\hline & Mean $\pm S D$ & $M e a n \pm S D$ \\
\hline \multicolumn{3}{|l|}{ Pre-Exercise Testing Data } \\
\hline Heart Rate (beats per minute) & $75 \pm 8$ & $77 \pm 11$ \\
\hline $\mathrm{VO}_{2}(\mathrm{~L} / \mathrm{min})$ & $3.5 \pm 0.7$ & $3.6 \pm 0.8$ \\
\hline Respiratory Quotient & $0.90 \pm 0.04$ & $0.88 \pm 0.05$ \\
\hline Systolic Blood Pressure (mm Hg) & $106 \pm 8$ & $106 \pm 11$ \\
\hline Diastolic Blood Pressure $(\mathrm{mm} \mathrm{Hg})$ & $71 \pm 8$ & $70 \pm 10$ \\
\hline Lactate $(\mathrm{mmol} / \mathrm{L})$ & $1.4 \pm 0.8$ & $1.1 \pm 0.3$ \\
\hline \multicolumn{3}{|l|}{ Exercise Testing Data } \\
\hline Anaerobic Threshold (\% of $\mathrm{VO}_{2}$ peak) & $65 \pm 5$ & $66 \pm 3$ \\
\hline Peak Heart Rate (beats per minute) & $178 \pm 12$ & $184 \pm 11$ \\
\hline Peak Power Output (Watts) & $183 \pm 48$ & $185 \pm 50$ \\
\hline Peak Aerobic Power $\left(\mathrm{VO}_{2} ; \mathrm{ml} / \mathrm{kg} / \mathrm{min}\right)$ & $38 \pm 10$ & $38 \pm 10$ \\
\hline Peak Lactate (mmol/L) & $11.9 \pm 3.2$ & $13.6 \pm 3.4$ \\
\hline Peak Respiratory Quotient & $1.18 \pm 0.06$ & $1.19 \pm 0.05$ \\
\hline Exercise Test Duration (Minutes) & $11.7 \pm 1.7$ & $12.3 \pm 1.6$ \\
\hline
\end{tabular}


Figure 1: Changes in Systolic Blood Pressure Following Remote Ischemic

Preconditioning (remote IPC). Individual (solid lines) and mean sample (dotted line) pre-post intervention changes are highlighted.

Figure 2: Changes in Peak Exercise Performance Following Remote Ischemic Preconditioning (remote IPC). Individual (solid lines) and mean sample (dotted line) pre-post intervention changes are highlighted. Abbreviations: oxygen consumption, $\mathrm{VO}_{2} ;$ milliliters $/ \mathrm{kilogram} /$ minute, $\mathrm{ml} / \mathrm{kg} / \mathrm{min}$. 


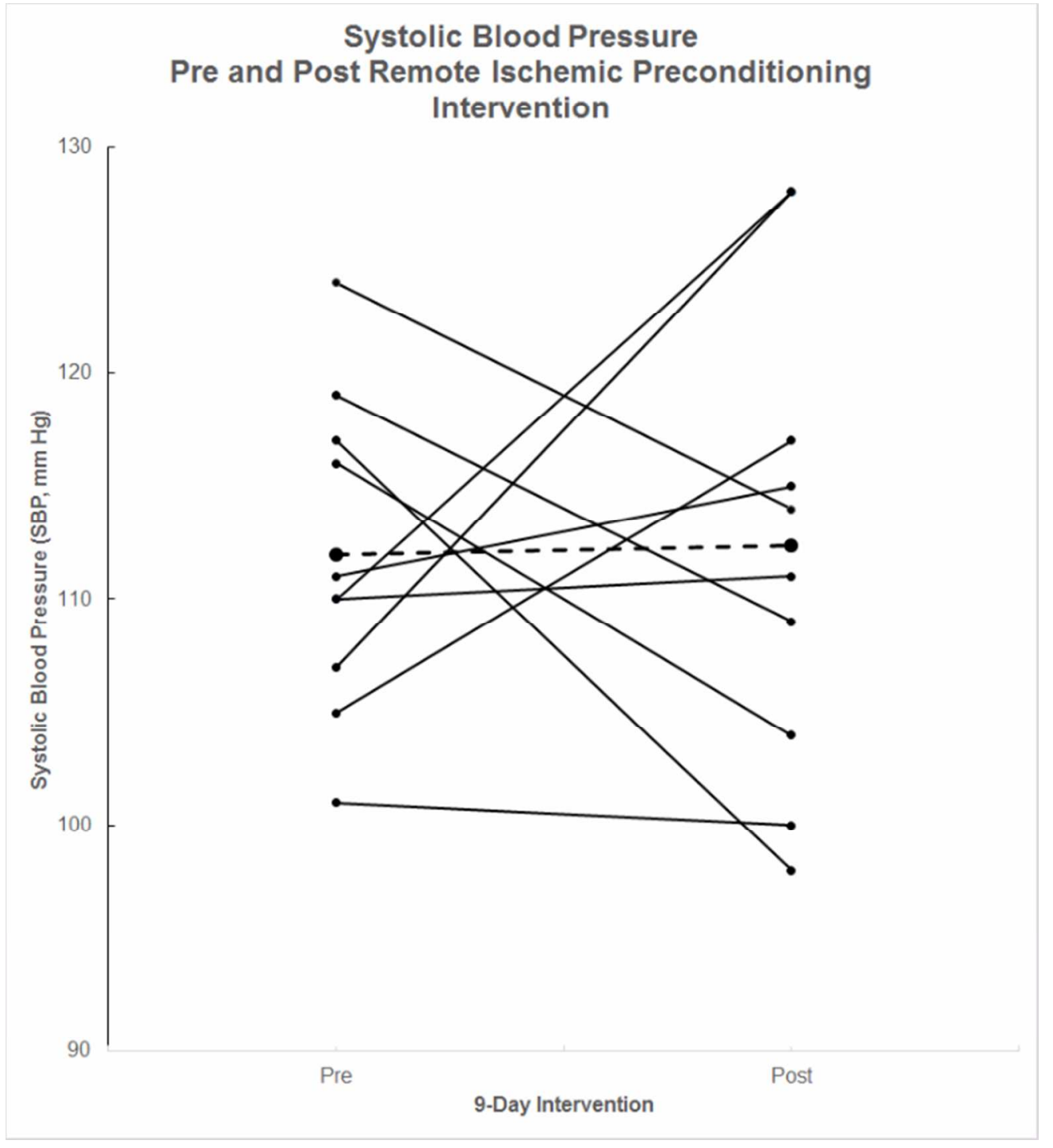

Figure 1: Changes in Systolic Blood Pressure Following Remote Ischemic Preconditioning (remote IPC). Individual (solid lines) and mean sample (dotted line) pre-post intervention changes are highlighted.

$61 \times 67 \mathrm{~mm}(300 \times 300 \mathrm{DPI})$ 


\section{Peak Oxygen Consumption Pre and Post Remote Ischemic Preconditioning Intervention}

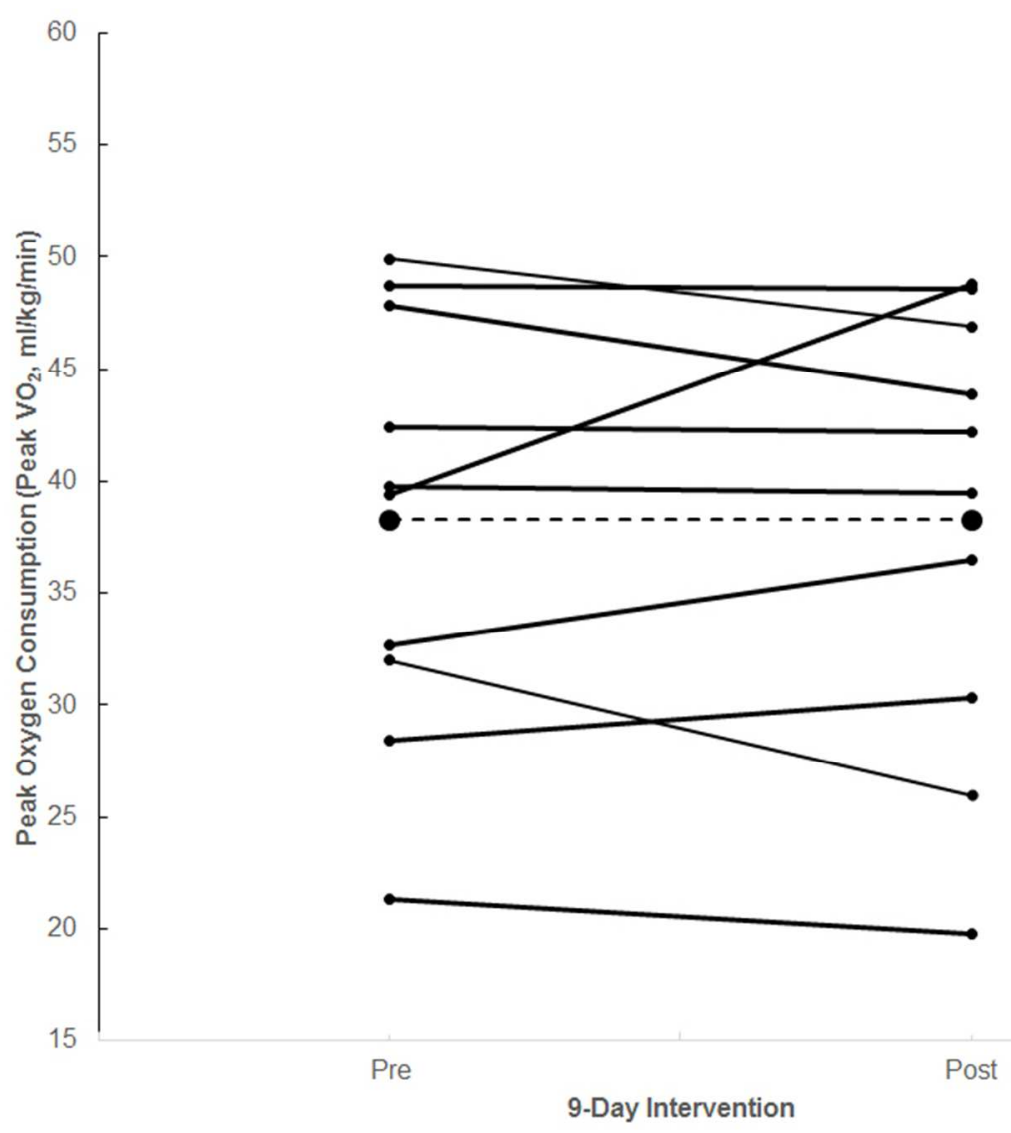

Figure 2: Changes in Peak Exercise Performance Following Remote Ischemic Preconditioning (remote IPC). Individual (solid lines) and mean sample (dotted line) pre-post intervention changes are highlighted. Abbreviations: oxygen consumption, VO2; milliliters/kilogram $/ \mathrm{minute}, \mathrm{ml} / \mathrm{kg} / \mathrm{min}$. $67 \times 67 \mathrm{~mm}(300 \times 300 \mathrm{DPI})$ 